-Research Highlight

\title{
Antipsychotic drug research and resting-state brain activity in normal adults
}

\author{
Yuan-Ye Ma \\ State Key Laboratory of Brain and Cognitive Science, Kunming Institute of Zoology, Chinese Academy of Sciences, \\ Kunming 650223, China
}

Corresponding author: Yuan-Ye Ma. E-mail: yuanma0716@vip.sina.com

(c) Shanghai Institutes for Biological Sciences, CAS and Springer-Verlag Berlin Heidelberg 2013

In the field of functional MRI, compared to observations of task-related brain activity, a growing number of studies have shown that spontaneous brain activity during the resting state may be more sensitive to defects in the cognitive functions of our brain.

In this issue, Zhu et al. ${ }^{[1]}$ report that methylphenidate (MPH), one of the most commonly-used stimulants for the treatment of attention deficit hyperactivity disorder (ADHD), induces stronger regional homogeneity ( $\mathrm{ReHo})$ in the left middle and superior temporal gyrus and weaker ReHo in the left lingual gyrus in normal male adults.

This study reveals several insights. First of all, knowledge of the effects of drugs on the default network contributes to our understanding of the mechanism underlying the action of this network, because the pharmacological effects of many drugs are relatively clear. Such studies prompt questions like: What do cognitive tasks and resting state have in common? ReHo changes in many psychiatric diseases, and abnormal ReHo has been observed in the resting state in ADHD, schizophrenia, Alzheimer's disease, Parkinson's disease, and major depressive disorder ${ }^{[2-5]}$. This further encourages the consideration of the functions of the default network that might be important for understanding mental diseases ${ }^{[2]}$.

Clinically, it is very difficult for patients with mental disorders, especially schizophrenia, to perform tasks during an $\mathrm{fMRI}$ scan. Another problem is that different participants may use different strategies to complete a task, and if so, it is conceivable that the results differ greatly even if partici- pants in the same group perform the same task. So, interpreting the results becomes difficult. However, it is much easier to scan patients in the resting state.

More importantly, this study was carried out in normal adults, not patients, and the results show that "the ReHo in some brain areas changes with MPH compared to placebo in normal adults, even though there are no behavioral differences. This method can be applied to patients with mental illness who may be treated with $\mathrm{MPH}$, and be used to compare the difference between patients taking $\mathrm{MPH}$ and normal participants, to help reveal the mechanism of how MPH works."

\section{REFERENCES}

[1] Zhu Y, Gao B, Hua J, Liu W, Deng Y, Zhang L, et al. The effects of methylphenidate on resting-state brain activity in normal adults: An fMRI study. Neurosci Bull 2013, 29: 16-27.

[2] Buckner RL, Andrews-Hanna JR, Schacter DL. The brain's default network: anatomy, function, and relevance to disease. Ann N Y Acad Sci 2008, 1124: 1-38.

[3] Greicius MD, Srivastava G, Reiss AL, Menon V. Defaultmode network activity distinguishes Alzheimer's disease from healthy aging: evidence from functional MRI. Proc Natl Acad Sci U S A 2004, 101: 4637-4642.

[4] Kennedy DP, Redcay E, Courchesne E. Failing to deactivate: resting functional abnormalities in autism. Proc Natl Acad Sci U S A 2006, 103: 8275-8280.

[5] Lustig C, Snyder AZ, Bhakta M, O'Brien KC, McAvoy M, Raichle $M E$, et al. Functional deactivations: change with age and dementia of the Alzheimer type. Proc Natl Acad Sci U S A 2003, 100: 14504-14509. 